\title{
Effect of carbon emission regulations on transport mode selection under stochastic demand
}

\author{
K. M. R. Hoen • T. Tan • J. C. Fransoo • \\ G. J. van Houtum
}

Received: 25 May 2011/Accepted: 7 May 2012/Published online: 21 June 2012

(C) The Author(s) 2012. This article is published with open access at Springerlink.com

\begin{abstract}
More and more companies are paying attention to their carbon footprint beyond production emissions. In this work we consider a 'carbon-aware' company (either by choice or enforced by regulation) that is reconsidering the transport mode selection decision. Traditionally the trade-off has been between lead time (and corresponding inventory costs) and transportation costs but now emission costs come into the equation. We use a carbon emission measurement methodology based on real-life data and incorporate it into an inventory model. We consider the results for different types of emission regulation (including voluntary targets). We find that even though large emission reductions can be obtained by switching to a different mode, the actual decision depends on the regulation and non-monetary considerations, such as lead time variability.
\end{abstract}

Keywords Green supply chains - Carbon emissions · Inventory model · Transport mode selection

\section{Introduction}

Over the last few decades global warming has received increasing attention. In 1995 the Intergovernmental Panel on Climate Change (IPCC) published an assessment which states that the increase in greenhouse gas concentrations tends to warm the surface of the earth and leads to other climate changes (IPCC 1995). Greenhouse gases is a term which is used to refer to a collection of gases among which are carbon dioxide (or carbon), methane, and CFCs. The assessment of the IPCC was used to formulate an important international commitment to reduce greenhouse gas

K. M. R. Hoen $(\bowtie) \cdot$ T. Tan · J. C. Fransoo · G. J. van Houtum School of Industrial Engineering, Eindhoven University of Technology, P.O. Box 513, 5600 MB Eindhoven, The Netherlands e-mail: k.m.r.hoen@tue.nl 
emissions in 1997: the Kyoto protocol. Besides the reduction targets the protocol offers three market-based mechanisms to meet the targets: emissions trading, clean development mechanism and joint implementation. The European Union has already implemented an emissions trading scheme (EU ETS) for the energyintensive industries which currently account for almost $50 \%$ of Europe's carbon emissions (European Commission 2008). In an ETS the regulator sets the cap of emissions for a given year and issues allowances that match the cap. Companies obtain allowances, either for free or by buying them at an auction, and should have enough allowances at the end of the period to meet its emissions. As a result, a trading market for allowances is established and the market determines the price for emissions. In this research we focus on carbon dioxide emissions because it is an important greenhouse gas.

Currently, no comprehensive regulations exist that impact carbon emissions resulting from all types of transport. However, in January 2009 the European Commission published a directive to include aviation in the EU ETS. As of early 2012 the regulation covers the 27 EU member states and Iceland, Liechtenstein, and Norway (European Commission 2012). A study of the Organization for Economic Co-operation and Development (OECD 2002) predicted that, without appropriate action, the carbon emissions resulting from transport will be doubled by 2020 (compared with 1990). This is not in line with the emissions of other areas, such as industry or power generation, which show a decreasing trend over the same period. Since 1990 the energy efficiency of transport has been increasing and is likely to continue increasing (European Commision 2007). The increase in emissions is due to the increase in demand for transportation and the existing trend in increased energy efficiency is not sufficient to balance this. In the (near) future governments are likely to develop regulations which restrict the emissions originating from transport activities.

Two main reasons exist for companies to exert effort on carbon emission abatement: The first one is voluntary commitment, as a response to pressure from customer preferences, environmental groups, and initiatives such as the carbon disclosure project. The second reason is to respond to emission regulations. For transportation this is currently limited but likely to extend in the future. The most likely types of regulations are (1) a (carbon or diesel) tax, (2) inclusion in or creation of a separate ETS, or (3) a hard constraint on emissions. We formulate a transport mode selection model that is able to incorporate any of these three regulation types. The impact each of the regulation types would bring in on the selected transport mode and expected total cost is different, of course. We elaborate on this in Sect. 5.

Companies need to seek opportunities to decrease demand for high energy transport modes to reduce their emissions, or to reduce the costs incurred by emission regulation. We consider the possibility to reduce carbon emissions by selecting transport modes which generate lower emissions per km traveled. We take the design of the supply chain as fixed, which we have also observed in practice to be the preferred problem scope by many companies. A company that has outsourced its transport activities is considered. Traditionally, the decision for a transport mode has been based on the lead time and transport cost trade-off, i.e. the longer the lead time, the higher the inventory required to deal with demand uncertainty, and the 
lower the transport cost. We extend this trade-off by including carbon emissions. Exactly one transport mode is selected. We consider a single product setting in order to focus on the interactions and the trade-offs between the relevant costs and carbon emissions.

We distinguish three contributions of our work. First, we formulate a transport mode selection model which analyzes the trade-off between inventory, transport, and emission costs for transport modes. Second, we use a methodology based on empirical data to obtain accurate estimates for the carbon emissions for different modes of transport and specific properties of the product. Finally, we investigate the effect of different types of regulations with respect to emissions on the selected transport mode and the corresponding emissions.

The outline of the remainder of the paper is as follows. First, the related literature is described in Sect. 2. Our model and the transport mode selection problem incorporating emissions are described in Sect. 3. In Sect. 4, we analyze the transport mode selection problem. In Sect. 5, we conduct a numerical study, using the NTM methodology, to determine the impact of product characteristics on the solution and the impact of different types of emission regulation. In Sect. 6, we draw conclusions.

\section{Related literature}

Both the operations management and transport literature, and specifically literature that incorporates carbon emissions, are related to this research. We next discuss each of these related fields briefly. Within the operations management field, literature on green supply chain management (GSCM) is connected to our work. GSCM is defined by Srivastava (2007) as "integrating environmental thinking into supply chain management including product design, material sourcing and selection, manufacturing processes, delivery of the final product to the consumers as well as end-of-life management of the product after its useful life". Overviews of GSCM literature are given by Corbett and Kleindorfer (2001a, b), Kleindorfer et al. (2005), Srivastava (2007), Sasikumar and Kannan (2009), and Gupta and Lambert (2008). Reverse logistics/ closed-loop supply chains is an important part of green supply chain management and a lot of research has already been conducted in this field; see for example Blumberg (2005) and Pochampally et al. (2009). Literature reviews of the field are given by Atasu et al. (2008) and Fleischmann et al. (1997).

The field of GSCM including carbon emissions is rapidly extending to include green inventory models that link the inventory and ordering behavior and emissions, Bonney and Jaber (2011) elaborate on this development. In Hua et al. (2011) a producer for which production emissions are bound by emission regulation is deciding how much to invest in technology to abate variable emissions in a profitmaximizing setting. Benjaafar et al. (2010) consider both emissions from production, transport and inventory in a lot-sizing problem. They consider several emission regulation policies and determine how they impact the operational lot-sizing decision. The emission abatement option they consider is to ship/produce more products at once to decrease the share of fixed emissions. Lastly, Rosič and 
Jammernegg (2010) consider a Newsvendor framework in which two supply sources are available, onshore and offshore, and transport emissions from the offshore source are bound by emission regulation. Ordering less from the offshore supplier is the emission abatement option considered.

Although these papers incorporate carbon emissions in inventory decisions, the transport modality is assumed to be an external parameter. Our work contributes to the literature in this field by considering and optimizing for the transport modality decision and inventory policy simultaneously. Moreover, we model the transport cost and emissions as a function of product characteristics.

Within the transport literature, several articles are related to our work. One paper that does take carbon emissions into account explicitly in decision making is Bauer et al. (2009) albeit in a different problem setting. The focus is on determining the planning for a rail service network and an integer linear programming formulation is developed to determine the service network design that minimizes the emissions. Our work differs since we focus on a production company that uses outsourced transport and explicitly take the impact on inventory into account.

Moreover, the works on transport mode selection are related. Most works provide a very detailed description of the transport activity and inventory is of minor importance. For our work, the transport mode selection articles that focus on the inventory-theoretic framework are relevant (see Tyworth 1991) for a literature review. This topic is covered by a vast body of literature and it was studied for the first time by Baumol and Vinod (1970). Recently, the field has been extending to take into account environmental effects. Several articles that aim at moving from high-energy transport modes (such as air and road) to low-energy transport modes are available. For example, Blauwens et al. (2006) investigate the effect of policy measures on modal shift, and the aim is to move away from road transport because of congestion. In Kiesmüller et al. (2005) the added value of using a slow mode in addition to a fast mode is considered.

Nevertheless, to the best of our knowledge the literature on transport mode selections problems does not quantify emissions and explicitly take them into account in decision making. Our work contributes to the transport mode selection literature by investigating the impact of emission regulation on the transport mode selection decision.

\section{Model description}

In this section, we formulate our model and the transport mode selection problem, in which the transport mode which minimizes the expected total cost, including an emissions cost, is selected. We consider a single production facility of a company that uses a single product as input to a production process. The production facility orders the product from a (possibly internal) supplier and several (two or more) transport modes are available for transport. The company determines on a regular basis, e.g. yearly, which transport mode to use for the product. They send out a request-for-quotation to a third-party logistics service providers (3PL) and, based on the offers they obtain, exactly one mode is selected which is used for all transport. 
When considering outsourced transport emissions a complicating factor is that the 'ownership' of the emissions is less clear: the shipper, or the logistics service provider. We assume that the shipper is solely responsible for the emissions resulting from transporting the items [even though they correspond to 'Scope 3' emissions of the Greenhouse Gas (GHG) Protocol]. This assumption is justified because the shipper creates the demand for transport. Moreover, it is in the best interest of the logistics provider to make the execution of the transport as efficient as possible, because emissions are aligned with fuel costs.

The accurate measurement of carbon emissions is an essential requirement to ensure that the emission targets are met and for companies to reduce their carbon emissions. The most well-known methodology is probably the GHG Protocol (2011). For this research, the network for transport and environment (NTM) method is selected because it provides a higher level of detail and the methodology provides estimates for parameter values for transport in Europe that are unknown to a company or logistics service provider. NTM is also involved with the European Committee for Standardization (CEN) in developing the European standard for emission calculation (NTM 2011).

In the model an emission cost is included, which represents one of the (self-) regulation types described in Sect. 1. The production facility orders each period under an infinite horizon. We assume that the selected transport mode is used for a sufficient number of periods such that an infinite horizon model is a valid approximation. For ease of reference, Table 1 represents all parameters of the model.

Table 1 Overview of product parameters (a) and mode specific parameters (b)

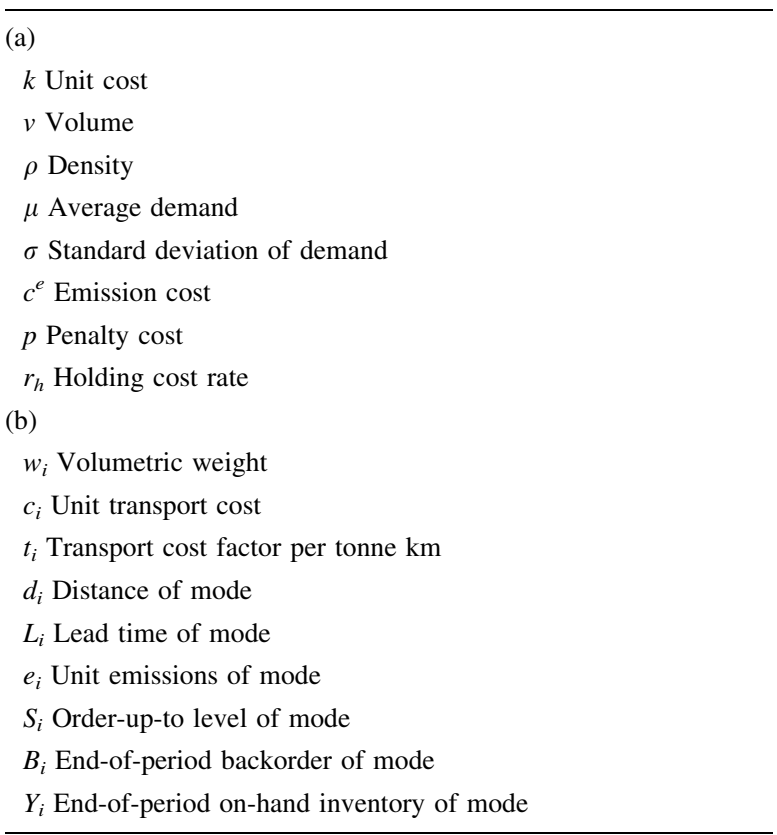


Several transport modes are available to ship the product, denoted by $I=\{1, \ldots, m\}(i \in I)$. The unit cost of the product, the product volume, and the product density are denoted by $k(k>0), v(v>0)$ and $\rho(\rho>0)$, respectively. Logistics service providers charge their customers based on the dimensional or volumetric weight of the shipment (NTM Air 2008; NTM Road 2008) to account for the fact that the volume is restricting for low-density products. The volumetric weight is determined by the following formula:

$$
w_{i}=v \max \left\{\rho, \bar{\rho}_{i}\right\}
$$

where $\bar{\rho}_{i}\left(\bar{\rho}_{i} \geq 0\right)$ is the minimum density, specified by the 3PL for mode $i$. Please note that if $\bar{\rho}_{i}=0$, then the actual weight of the product is always used to determine the transport cost of mode $i$.

Let $c_{i}\left(c_{i}>0\right)$ denote the unit transportation cost for mode $i$ charged by the 3PL which is based on the volumetric weight of the product and a cost factor per kilogram of product shipped over $1 \mathrm{~km}(€ / \mathrm{kg}, \mathrm{km}) t_{i}: c_{i}=d_{i} t_{i} w_{i}$, where $d_{i}$ is the distance in $\mathrm{km}$ when mode $i$ is used. The deterministic supply lead time for mode $i$ is denoted by $L_{i}\left(L_{i} \in \mathbb{N}_{0}\right.$, where $\left.\mathbb{N}_{0}=\mathbb{N} \cup\{0\}\right)$. We assume that $L_{i}$ is increasing in $d_{i}$. We assume that the supplier holds sufficient stock to satisfy the demand within the specified lead time.

Demand per period for the product follows a continuous distribution and is characterized by mean $\mu$ and standard deviation $\sigma(\mu, \sigma>0)$. Demands in different periods are assumed to be independent and identically distributed (i.i.d.). Demand during lead time plus the review period is characterized by mean $\mu_{i}=\left(L_{i}+1\right) \mu$ and standard deviation $\sigma_{i}=\sqrt{L_{i}+1} \sigma$ (follows from the i.i.d. assumption). Let $f_{i}(x)$ and $F_{i}(x)$ denote the probability density function and cumulative distribution function of demand during $L_{i}+1$ periods.

We consider a variable emission factor but no fixed emission factor per shipment, because transport is outsourced to a 3PL and the shipper has no control over the actual shipping. We assume, in accordance with NTM, that each product is shipped with an averagely loaded vehicle. The two steps in determining the emissions for a unit transported with a specific vehicle type are: determine the emissions for a vehicle with an average load and then allocate the emissions of the averagely-loaded vehicle to one unit of the product, based on weight. Let $e_{i}$ denote the emissions associated with shipping one unit of the product with mode $i$ :

$$
e_{i}=\left(E_{i}+\epsilon_{i} d_{i}\right) \frac{w_{i}}{\hat{W}_{i} \cdot \lambda_{i}},
$$

where $E_{i}$ is a constant emission factor, $\epsilon_{i}$ is a variable emission factor per $\mathrm{km}, \hat{W}_{i}$ is the maximum load of the vehicle, and $\lambda_{i}$ is the average load factor of the vehicle. The load factor of a vehicle is defined as the actual load divided by the maximum load. Let $c^{e}\left(c^{e} \geq 0\right)$ denote the emission cost per unit of emissions (e.g. tonne).

When an item is required at the facility and there are no units on stock, the demand is backordered. A penalty cost $p(p>0)$ is incurred per unit on backorder at the end of a period. The average number of items backordered at the end of a period for mode $i$ is denoted by $\mathbb{E}\left[B_{i}\left(c^{e}\right)\right]$. We write this variable as a function of emission cost $c^{e}$ for later purposes. The holding costs $h_{i}$ are defined as a function of 
the holding cost rate $r_{h}\left(r_{h}>0\right)$ of the opportunity cost associated with one product $\left(k+c_{i}+c^{e} e_{i}\right): h_{i}\left(c^{e}\right)=r_{h}\left(k+c_{i}+c^{e} e_{i}\right)$. Holding costs are incurred for each unit on stock at the end of a period, and the average is denoted by $\mathbb{E}\left[Y_{i}\left(c^{e}\right)\right]$.

The objective function, the expected total cost per period, is denoted by $C_{i}\left(c^{e}\right)$, for transport mode $i$ and emission $\operatorname{cost} c^{e}$, which consists of a penalty cost, holding cost, and transportation cost.

$$
C_{i}\left(c^{e}\right)=p \mathbb{E}\left[B_{i}\left(c^{e}\right)\right]+h_{i}\left(c^{e}\right) \mathbb{E}\left[Y_{i}\left(c^{e}\right)\right]+\left(c_{i}+c^{e} e_{i}\right) \mu
$$

The average number of units shipped per period is equal to the average demand $\mu$.

In each period first an order is placed (and earlier placed orders may arrive) and after that demand occurs. We assume that an order-up-to policy, or a base-stock policy, is used and we optimize the performance for this policy. Let $S_{i}$ denote the order-up-to level for transport mode $i$. The optimal order-up-to level coincides with the solution of the Newsvendor problem (Axsäter 2006). The expected cost per period for mode $i$, given order-up-to level $S_{i}\left(C_{i}\left(S_{i} \mid c^{e}\right)\right)$, is then:

$$
C_{i}\left(S_{i} \mid c^{e}\right)=h_{i}\left(c^{e}\right)\left(S_{i}-\mu_{i}\right)+\left(p+h_{i}\left(c^{e}\right)\right) G_{i}\left(S_{i}\right)+\left(c_{i}+c^{e} e_{i}\right) \mu,
$$

where $G_{i}(y)=\int_{y}^{\infty}(x-y) f_{i}(x) \mathrm{d} x$.

We end this section with the description of the cost-minimization problem $\left(\mathcal{C}_{i}\right)$ and transport mode selection problem $(\mathcal{C})$ in which we set the cost-minimizing order-up-to level and select a transport mode accordingly. Let the cost-minimization problem for transport mode $i$ be defined as follows:

$$
C_{i}^{*}\left(c^{e}\right)=\min _{S_{i}} C_{i}\left(S_{i} \mid c^{e}\right)
$$

The mode that minimizes the minimum total expected cost is selected in the transport mode selection problem (TMSP):

$$
(\mathcal{C}) \quad \min _{i \in I} C_{i}^{*}\left(c^{e}\right) .
$$

\section{Analysis}

In this section, we first solve the cost-minimization problem $\left(\mathcal{C}_{i}\right)$, for a given transport mode, and we solve the TMSP $(\mathcal{C})$ for a given emission cost value in Sect. 4.1. Then we derive conditions a mode must meet to be selected for at least one $c^{e}$ value, and derive the corresponding range of $c^{e}$ for which that mode is selected in Sect. 4.2. In Sect. 4.3 a special case with Normally distributed demand is described. The proofs are given in Appendix 1.

\subsection{The cost-minimization and transport mode selection problem}

The optimal order up-to level $\left(S_{i}^{*}\left(c^{e}\right)\right)$, for mode $i$ and emission cost $c^{e}$, follows from a direct application of the result of a single-period Newsvendor problem [see e.g. Axsäter (2006)], and satisfies the following equation: 


$$
F_{i}\left(S_{i}^{*}\left(c^{e}\right)\right)=\frac{p}{p+h_{i}\left(c^{e}\right)},
$$

The cost associated with the optimal order-up-to level for mode $i$ and emission cost $c^{e}\left(C_{i}^{*}\left(c^{e}\right)\right)$ is:

$$
C_{i}^{*}\left(c^{e}\right)=h_{i}\left(c^{e}\right)\left(F_{i}^{-1}\left(\alpha_{i}\left(c^{e}\right)\right)-\mu_{i}\right)+\left(p+h_{i}\left(c^{e}\right)\right) G_{i}\left(F_{i}^{-1}\left(\alpha_{i}\left(c^{e}\right)\right)\right)+\left(c_{i}+c^{e} e_{i}\right) \mu,
$$

where $\alpha_{i}\left(c^{e}\right):=\frac{p}{p+h_{i}\left(c^{e}\right)}$. For brevity reasons, let $C_{i, 0}^{*}=C_{i}^{*}(0)$. The average backorder and the on-hand inventory at the end of a period associated with the optimal order-upto level $\left(\mathbb{E}\left[B_{i}^{*}\left(c^{e}\right)\right]\right.$ and $\left.\mathbb{E}\left[Y_{i}^{*}\left(c^{e}\right)\right]\right)$ are determined by the following equations:

$$
\begin{aligned}
& \mathbb{E}\left[B_{i}^{*}\left(c^{e}\right)\right]=G_{i}\left(S_{i}^{*}\left(c^{e}\right)\right) \\
& \mathbb{E}\left[Y_{i}^{*}\left(c^{e}\right)\right]=S_{i}^{*}\left(c^{e}\right)-\mu_{i}+G_{i}\left(S_{i}^{*}\left(c^{e}\right)\right) .
\end{aligned}
$$

Using these equations, we derive how an increase in the emission cost impacts the model.

Proposition 1 The following monotonicity results hold for the model:

(a) $S_{i}^{*}\left(c^{e}\right)$ is decreasing in $c^{e}$,

(b) $\mathbb{E}\left[B_{i}^{*}\left(c^{e}\right)\right]$ is increasing in $c^{e}$,

(c) $\mathbb{E}\left[Y_{i}^{*}\left(c^{e}\right)\right]$ is decreasing in $c^{e}$,

(d) $C_{i}^{*}\left(c^{e}\right)$ is increasing in $c^{e}$.

This implies that if the emission cost increases, the order-up-to level decreases because inventory is more expensive. Hence, the average end of period on-hand inventory decreases and the average backorder increases. As a net result, the total average period costs increase. Note that the monotonicity results also apply to $k, v, \rho$, and $d_{i}$, since $h_{i}\left(c^{e}\right)$ is increasing in those variables.

We now derive the solution to the TMSP. Let $C^{*}\left(c^{e}\right)$ denote the optimal value of the minimum total expected cost, then $C^{*}\left(c^{e}\right)=C_{j}^{*}\left(c^{e}\right)$ for $j=\arg \min _{i \in I} C_{i}^{*}\left(c^{e}\right)$ for a given $c^{e}$ value. The solution to Problem $(\mathcal{C})$ is to select mode $j$ (with order-up-to level $S_{j}^{*}\left(c^{e}\right)$ ). If mode $j$ is selected for at least one $c^{e}$ value, we state that mode $j$ is a preferred mode.

\subsection{The impact of the emission cost on the solution}

We now specify two conditions mode $i_{2} \in I$ must meet to be a preferred mode. First of all, mode $i_{2}$ is not a preferred mode if there is a mode $i_{1} \in I$ such that $C_{i_{1}, 0}^{*}<C_{i_{2}, 0}^{*}$ and $e_{i_{1}}<e_{i_{2}}$, i.e. mode $i_{2}$ should be efficient in terms of minimum total expected cost for $c^{e}=0$ and unit emissions. This implies that if mode $i_{1}$ is strictly less polluting and results in strictly lower costs than mode $i_{2}$ for $c^{e}=0$, it is less polluting and cheaper for all values of the emission cost.

Let us now assume that the remaining modes are ordered in decreasing $e_{i}$ and in increasing $C_{i, 0}^{*}$ values. It then holds that the modes that minimize $C_{i, 0}^{*}$ and $e_{i}$ are preferred modes (they are selected for at least $c^{e}=0$ and $c^{e}=\infty$, respectively), i.e. 
the cheapest mode is selected for low emission cost values and the greenest mode for (very) high values. If there is one mode that minimizes both, then it is the only preferred mode.

For the modes that do not minimize $e_{i}$ or $C_{i, 0}^{*}$ efficiency is not sufficient to guarantee that mode $i$ is a preferred mode, i.e. that it is selected for at least one $c^{e}$ value. Consider three efficient modes $i_{1}, i_{2}, i_{3} \in I$ such that $C_{i_{1}, 0}^{*} \leq C_{i_{2}, 0}^{*} \leq C_{i_{3}, 0}^{*}$ and $e_{i_{1}} \geq e_{i_{2}} \geq e_{i_{3}}$. If mode $i_{2}$ has marginally lower emissions than mode $i_{1}$ and significantly larger costs $\left(C_{i_{2}, 0}^{*}\right.$ close to $\left.C_{i_{3}, 0}^{*}\right)$, then mode $i_{2}$ may not be a preferred mode. For our model we determine numerically which modes are preferred modes, but in the special case in Sect. 4.3 the second condition mode $i_{2}$ must meet to be a preferred mode is written explicitly. Let $\mathbb{I}=\{1,2, \ldots, \mathcal{M}\}$ denote the set of preferred modes and let us renumber the preferred modes such that they are ordered in decreasing $e_{i}$ and increasing $C_{i, 0}^{*}$ values.

We now derive the range of emission cost values for which mode $i$ is selected. For a pair of transport modes $i, i+1 \in \mathbb{I}$, let $c_{i, i+1}^{e}$ denote the value of the emission cost for which the minimum average period costs are equal $\left(C_{i}^{*}\left(c_{i, i+1}^{e}\right)=\right.$ $\left.C_{i+1}^{*}\left(c_{i, i+1}^{e}\right)\right)$. We refer to this value as the indifference emission cost for modes $i$ and $i+1$. For emission cost values less than the indifference cost, $C_{i}^{*}\left(c^{e}\right)<C_{i+1}^{*}\left(c^{e}\right)$ (and vice versa). This result follows directly from the ordering of the modes in $\mathbb{I}$. As a result, transport mode $i$ is the selected transport mode for $c^{e} \in\left[c_{i-1, i}^{e}, c_{i, i+1}^{e}\right]$, where $c_{0,1}^{e}=0$ and $c_{\mathcal{M}, \mathcal{M}+1}^{e}=\infty$, for $i-1, i, i+1 \in \mathbb{I}$. For the model with a general demand distribution, $c_{i, i+1}^{e}$ can only be determined numerically. In the special case of our model in Sect. 4.3, a closed-form expression is derived.

In ecological economics, marginal analysis is used to determine the effect of emission regulation on emission abatement effort (Pindyck and Rubinfeld 2008). In marginal analysis it is assumed that companies incur costs to reduce emissions and that these costs are in general increasing in the emission reductions. If a carbon tax is set to a value of $\tilde{c}^{e}$ per unit of emissions, all companies will reduce their emissions until the point where the marginal cost of reduction is higher than the tax. It is then cheaper to pay the tax for the remaining emissions than to decrease the emissions further. In our case the emission reduction option is to switch to a less polluting mode. Hence, following the same logic, if $\tilde{c}^{e} \in\left[c_{i-1, i}^{e}, c_{i, i+1}^{e}\right]$, i.e. the actual emission price is higher than the cost increase (per unit of emissions reduced) due to using mode $i$ compared to mode $i-1$, then transport mode $i$ is used.

\subsection{Special case: normally distributed demand}

To be able to derive closed-form expressions, we assume that demand follows a normal distribution. Let the $p d f$ and the $c d f$ of single-period demand be denoted by $\phi(x)$, and $\Phi(x)$, respectively. The minimum expected total cost for mode $i$ and emission $\operatorname{cost} c^{e}$ is then:

$$
C_{i}^{*}\left(c^{e}\right)=\sqrt{L_{i}+1} \sigma\left(p+h_{i}\left(c^{e}\right)\right) \phi\left(\Phi^{-1}\left(\alpha_{i}\left(c^{e}\right)\right)\right)+\left(c_{i}+c^{e} e_{i}\right) \mu .
$$

It holds that $C_{i}^{*}\left(c^{e}\right)$ is increasing in $v, \rho$, and $d_{i}$ (using the fact that $L_{i}$ is increasing in $d_{i}$ ), a direct application of the result of Proposition 1 . 
To be able to derive an explicit condition that specifies whether a mode is a preferred mode or not and a closed-form expression for $c_{i, i+1}^{e}$, we consider a model in which the holding costs only depend on the unit cost of the product: $h=r_{h} k$, which is then equal for all transport modes, as is $\alpha$. We believe that this approximation is relatively accurate because the impact of $c^{e}$ on the holding costs is relatively low, especially for high $k$ values. The minimum expected total cost is then:

$$
C_{i}^{*}\left(c^{e}\right)=\sqrt{L_{i}+1} \sigma(p+h) \phi\left(\Phi^{-1}(\alpha)\right)+\left(c_{i}+c^{e} e_{i}\right) \mu .
$$

where $\alpha:=\frac{p}{p+h}$. In the next lemma we formally derive a condition mode $i_{2} \in I$ must meet to be preferred:

Lemma 1 Consider transport modes $i_{1}, i_{2}, i_{3} \in I$ such that $C_{i_{1}, 0}^{*} \leq C_{i_{2}, 0}^{*} \leq C_{i_{3}, 0}^{*}$, and $e_{i_{1}} \geq e_{i_{2}} \geq e_{i_{3}}$.

(a) If $e_{i_{2}}>\bar{e}_{i_{2}}\left(i_{1}, i_{3}\right)$, then transport mode $i_{2}$ is not selected for any $c^{e} \geq 0\left(C_{i_{2}}^{*}\left(c^{e}\right)>\min \left\{C_{i_{1}}^{*}\left(c^{e}\right), C_{i_{3}}^{*}\left(c^{e}\right)\right\}\right)$,

(b) If $e_{i_{2}} \leq \bar{e}_{i_{2}}\left(i_{1}, i_{3}\right)$, then $C_{i_{2}}^{*}\left(c^{e}\right)=\min \left\{C_{i_{1}}^{*}\left(c^{e}\right), C_{i_{2}}^{*}\left(c^{e}\right), C_{i_{3}}^{*}\left(c^{e}\right)\right\}$ for $c^{e} \in$ $\left[c_{i_{1}, i_{2}}^{e}, c_{i_{2}, i_{3}}^{e}\right]$,

where

$$
\bar{e}_{i_{2}}\left(i_{1}, i_{3}\right)=e_{i_{1}}+\left(e_{i_{3}}-e_{i_{1}}\right) \frac{C_{i_{2}, 0}^{*}-C_{i_{1}, 0}^{*}}{C_{i_{3}, 0}^{*}-C_{i_{1}, 0}^{*}} .
$$

The interpretation of this lemma is that mode $i_{2}$ needs to outperform the convex combination of modes $i_{1}$ and $i_{3}$ in terms of $e_{i}$ and $C_{i, 0}^{*}$. As a result, the modes in $\mathbb{I}$, in terms of $e_{i}$ and $C_{i, 0}^{*}$, form a convex hull. The following corollary states the necessary and sufficient condition for mode $i$ to be preferred (follows from Lemma $1 \mathrm{~b}$ ).

Corollary 1 If transport mode $i_{2}$ is a preferred transport mode $\left(i_{2} \in \mathbb{I}\right)$, then $e_{i_{2}} \leq \bar{e}_{i_{2}}\left(i_{1}, i_{3}\right)$ for all $i_{1}<i_{2}<i_{3}$ and $i_{1}, i_{3} \in \mathbb{I}$. Transport mode $i_{2}$ is selected for $c^{e} \in\left[c_{i_{2}-1, i_{2}}^{e}, c_{i_{2}, i_{2}+1}^{e}\right]$, where $c_{0,1}^{e}=0$ and $c_{\mathcal{M}, \mathcal{M}+1}^{e}=\infty$.

Consider modes $i, i+1 \in \mathbb{I}$ (hence $e_{i} \geq e_{i+1}$ and $C_{i, 0}^{*} \leq C_{i+1,0}^{*}$ ), then the closed form expression for the indifference $\operatorname{cost} c_{i, i+1}^{e}$ is:

$$
c_{i, i+1}^{e}=\frac{c_{i}-c_{i+1}-\frac{\sigma}{\mu}\left(\sqrt{L_{i+1}+1}-\sqrt{L_{i}+1}\right)(p+h) R}{e_{i+1}-e_{i}},
$$

where $R=\phi\left(\Phi^{-1}(\alpha)\right)$. Below we investigate the effect of parameters on $c_{i, i+1}^{e}$. A parameter can be increased or decreased up to the point where $C_{i, 0}^{*}=C_{i+1,0}^{*}$. To obtain insight in the effect of parameters, we neglect the constant emission factor and minimum density. Rewriting Eq. (10) in terms of product and mode parameters yields:

$$
c_{i, i+1}^{e}=\frac{t_{i}-t_{i+1}-\frac{1}{v \rho} \frac{\sigma}{\mu} \frac{\zeta_{i+1}-\zeta_{i}}{\sqrt{d \zeta_{i+1}+1}+\sqrt{d \zeta_{i}+1}}(p+h) R}{\frac{\epsilon_{i+1}}{\hat{W}_{i+1} \lambda_{i+1}}-\frac{\epsilon_{i}}{\hat{W}_{i} \lambda_{i}}},
$$


where $L_{i}=\zeta_{i} \cdot d$ and $\zeta_{i}>0$ and we have used $\sqrt{a}-\sqrt{b}=\frac{a-b}{\sqrt{a}+\sqrt{b}}$. The effects described below hold when $L_{i+1}>L_{i}\left(\zeta_{i+1}>\zeta_{i}\right)$, when $L_{i+1}<L_{i}$ the effects change accordingly. Note that both the numerator and the denominator are negative.

Distance The indifference emission cost is decreasing in $d$. This implies that for larger distances cleaner modes are selected, because the inventory holding and penalty costs are balanced by lower transport and emission costs.

Weight An increase of the weight (volume or density) has a similar effect on $c_{i, i+1}^{e}$. So heavier products are shipped with cleaner modes. The reason is that the share of total costs due to penalty and holding costs decreases and hence favoring mode $i+1$.

Unit cost The indifference emission cost is increasing in unit cost $k$. For more expensive products, faster and more polluting modes are used. The reason is that the share of holding and penalty costs increases and hence favoring mode $i$ with a shorter lead time.

Demand variability When demand variability increases, the indifference emission cost increases. When demand variability is high a faster (and more polluting) mode is selected to cope with the uncertainty.

Penalty cost We have observed that the indifference emission cost is increasing in $p$. So when it is important that products are available when they are requested, a faster mode is selected.

\section{Numerical study}

In this section we conduct a numerical study to gain managerial insights from the TMSP. In Sect. 5.1 we illustrate the emission calculations for one particular vehicle/ vessel for each of the four transport mode types for a test bed. Next we analyze the effect of parameters on the solution to the TMSP in Sect. 5.2. We analyze the impact of different types of emission regulations for four specific products in Sect. 5.3.

\subsection{NTM emission calculations}

In this section we apply the NTM methodology (NTM 2011) to determine the unit carbon emissions for our numerical study. The NTM method specifies emissions for four transport types: air, road, rail, and water, which are described in detail in Appendix 2. We only consider single-mode transportation (terminal-terminal).

Let air, road, rail, and water transport be denoted by subscript 1, 2, 3, and 4, respectively. Table 2 presents for each modality the load factor, maximum capacity of the vehicle and the distance. Distance is expressed as a function of the road distance denoted by $d$. Note that for rail and water transport the maximum capacity includes the weight of the train/vessel. The corresponding unit emissions, as a function of $v, \rho$, and $d$ (in $\mathrm{kg} \mathrm{CO}$ per unit shipped) are then: 
Table 2 Transport parameter values

\begin{tabular}{llcl}
\hline Modality & $\begin{array}{l}\text { Load } \\
\text { factor (\%) }\end{array}$ & $\begin{array}{l}\text { Maximum } \\
\text { capacity (t) }\end{array}$ & Distance \\
\hline Air & 80 & 29 & $d_{1}=0.8 d$ \\
Road & 70 & 26 & $d_{2}=d$ \\
Rail & 50 & 1,000 & $d_{3}=d$ \\
Water & 50 & 3,840 & $d_{4}=1.2 d$ \\
\hline
\end{tabular}

$$
\begin{aligned}
& e_{1} \approx v \max \{167, \rho\}\left(1.783 \cdot 10^{-1}+5.295 d \cdot 10^{-4}\right), \\
& e_{2} \approx v \max \{250, \rho\}\left(3.214 \cdot 10^{-4}+4.836 d \cdot 10^{-5}\right), \\
& e_{3} \approx v \cdot \rho \cdot 2.223 d \cdot 10^{-5} \\
& e_{4} \approx v \cdot \rho \cdot 1.3904 d \cdot 10^{-5}
\end{aligned}
$$

For road transport, the constant emission factor corresponds with a part of the route that is traveled on urban routes (for the remainder we assume motor way). From Eq. (11) follows that, for the vehicles selected and the assumptions made, $e_{1} \geq e_{2} \geq e_{3} \geq e_{4} \forall v, \rho, d \geq 0$, i.e. the modes in decreasing order of emissions are air, road, rail, and water.

We now apply these equations to a test bed consisting of realistic values. We specify two parameter values, representing a low and high value: distance (800 and $3,000 \mathrm{~km})$, density $\left(100\right.$ and $\left.1,000 \mathrm{~kg} / \mathrm{m}^{3}\right)$ and volume $(1$ and $500 \mathrm{l})$ of the product. In total we have $2^{3}=8$ instances. Applying these values to Eq. (11) yields the unit emissions (in $\mathrm{kg} \mathrm{CO}_{2}$ ) in Table 3. Observe that there are large differences in emissions and in the ratio of the emissions of two modes. For air and road, it is in the range $8-15$, for road and rail $2-5$, and for rail and water always 1.6 . The ratio of air and water emissions is at most 100 . The reasons that the ratios differ are the constant emission factor and minimum density, for air and road transport. Hence, the ratio is highest for the small distance and low density (and smallest for the large distance and high density) instance. Table 3 also shows that the unit emissions can be as high as $883 \mathrm{~kg} \mathrm{CO}_{2}$ per unit shipped. If the carbon price is $€ 15 / \mathrm{t}$, the costs increase with up to $€ 15 \cdot 0.883=€ 13.25$ per unit, which can be a large part of the total costs depending on the value of the product.

\subsection{Results of the transport mode selection problem}

In this section we describe the effect of parameters on the indifference emission cost. We assume that demand follows a Normal Distribution to allow for fast calculation times. We assume that $L_{1}$ is fixed at 1 day and that $L_{2}, L_{3}$ and $L_{4}$ depend on the distance; the speed is 400,240 , and $160 \mathrm{~km} /$ day for road, rail and water, respectively. We select the test bed of Sect. 5.1 and we specify in addition the following parameter values: $\mu=10, \sigma=2, L_{1}=1, L_{2}=\frac{d}{400}, L_{3}=\frac{d}{240}, L_{4}=$ $\frac{1.2 d}{160}, t_{1}=3.125 \cdot 10^{-5}, t_{2}=1.250 \cdot 10^{-5}, t_{3}=1.000 \cdot 10^{-5}, t_{4}=7.500 \cdot 10^{-6}, r_{h}=$ $\frac{0.25}{300}$, and $p=10 r_{h} k$. We believe that these values are a good representation of reality. We have selected two values of $k$ that yield different results. For small 
Table 3 Unit emissions air, rail, road and water transport (in $\mathrm{kg} \mathrm{CO}_{2}$ )

\begin{tabular}{|c|c|c|c|c|c|c|c|c|c|}
\hline \multirow[t]{2}{*}{$\mathrm{v}(1)$} & \multirow{2}{*}{$\begin{array}{l}d(\mathrm{~km}) \\
\rho\left(\mathrm{kg} / \mathrm{m}^{3}\right)\end{array}$} & \multicolumn{4}{|l|}{800} & \multicolumn{4}{|l|}{3,000} \\
\hline & & $e_{1}$ & $e_{2}$ & $e_{3}$ & $e_{4}$ & $e_{1}$ & $e_{2}$ & $e_{3}$ & $e_{4}$ \\
\hline 1 & 100 & 0.101 & 0.010 & 0.002 & 0.001 & 0.295 & 0.036 & 0.007 & 0.004 \\
\hline 500 & 100 & 50.259 & 4.876 & 0.889 & 0.556 & 147.528 & 18.174 & 3.335 & 2.086 \\
\hline 1 & 1,000 & 0.602 & 0.039 & 0.018 & 0.011 & 1.767 & 0.145 & 0.067 & 0.042 \\
\hline 500 & 1,000 & 300.950 & 19.504 & 8.892 & 5.562 & 883.400 & 72.697 & 33.345 & 20.856 \\
\hline
\end{tabular}

Table 4 Indifference emission costs in $€ / t \mathrm{CO}_{2}$

\begin{tabular}{|c|c|c|c|c|c|c|c|c|}
\hline \multirow[t]{2}{*}{$d(\mathrm{~km})$} & \multirow[t]{2}{*}{$v(1)$} & \multirow{2}{*}{$\begin{array}{l}k \\
\rho\left(\mathrm{kg} / \mathrm{m}^{3}\right)\end{array}$} & \multicolumn{3}{|l|}{2,000} & \multicolumn{3}{|l|}{9,000} \\
\hline & & & $c_{1,2}^{e}$ & $c_{2,3}^{e}$ & $c_{3,4}^{e}$ & $c_{1,2}^{e}$ & $c_{2,3}^{e}$ & $c_{3,4}^{e}$ \\
\hline 800 & 1 & 100 & 2,091 & 26,079 & 507,780 & 9,441 & 118,102 & $2,285,429$ \\
\hline 800 & 500 & 100 & 0 & 0 & 896 & $11^{*}$ & - & 4,452 \\
\hline 800 & 1 & 1,000 & 321 & 9,784 & 50,670 & 1,507 & 44,357 & 228,435 \\
\hline 800 & 500 & 1,000 & 0 & 0 & 0 & 0 & 0 & 338 \\
\hline 3,000 & 1 & 100 & 3,469 & 15,111 & 281,594 & 15,649 & 68,751 & $1,267,591$ \\
\hline 3,000 & 500 & 100 & 0 & 0 & 444 & $10^{*}$ & - & 2,416 \\
\hline 3,000 & 1 & 1,000 & 533 & 5,685 & 28,052 & 2,476 & 25,914 & 126,652 \\
\hline 3,000 & 500 & 1,000 & 0 & 0 & 0 & 0 & 0 & 134 \\
\hline
\end{tabular}

values of $k$, water transport is the only preferred mode; therefore, we have selected two large values of $k(2,000$ and 9,000).

For our set of assumptions water transport is always a preferred mode but it may only be selected for very large values of $c^{e}$. In total, the test bed consists of 16 instances and the indifference emission cost $\left(c_{i, i+1}^{e}\right.$ in $\left.€ / \mathrm{CO}_{2}\right)$ is determined numerically for the instances; the results are in Table 4. In instances 2 and 6 there is a cell with $11^{*}$ and $10^{*}$, respectively, these numbers represent the indifference emission cost between air and rail transport $\left(c_{1,3}^{e}\right)$, because road transport is not efficient in those instances. In line with the effects described in Sect. 4, the indifference emission cost is decreasing in $v, \rho$, and $d$, and increasing in $k$. For air and road transport $c_{1,2}^{e}$ is increasing in $d$, because air transport is more attractive for large distances $\left(L_{1}=1\right.$ and the constant emission factor $E_{1}$ is significant).

We next examine the effect of several parameters on the indifference emission costs graphically. The same parameter values are used as before, except for the following: $k=5,000, v=100, \rho=500$, and $d=1,200$. Figure 1 shows how the indifference emission costs change as a function of product volume. We see from this graph that air transport is only selected for low volume products. If the volume is 1181 or more, it is never selected (because $C_{1,0}^{*}>C_{2,0}^{*}$ ). Road transport is never selected for $v \geq 450 l$. Recall that the distance between $c_{i-1, i}^{e}$ and $c_{i, i+1}^{e}$ indicates the range of $c^{e}$ for which mode $i$ is selected for volume $v$. If this range is large for a particular value, then this implies that the selected mode is relatively robust to 


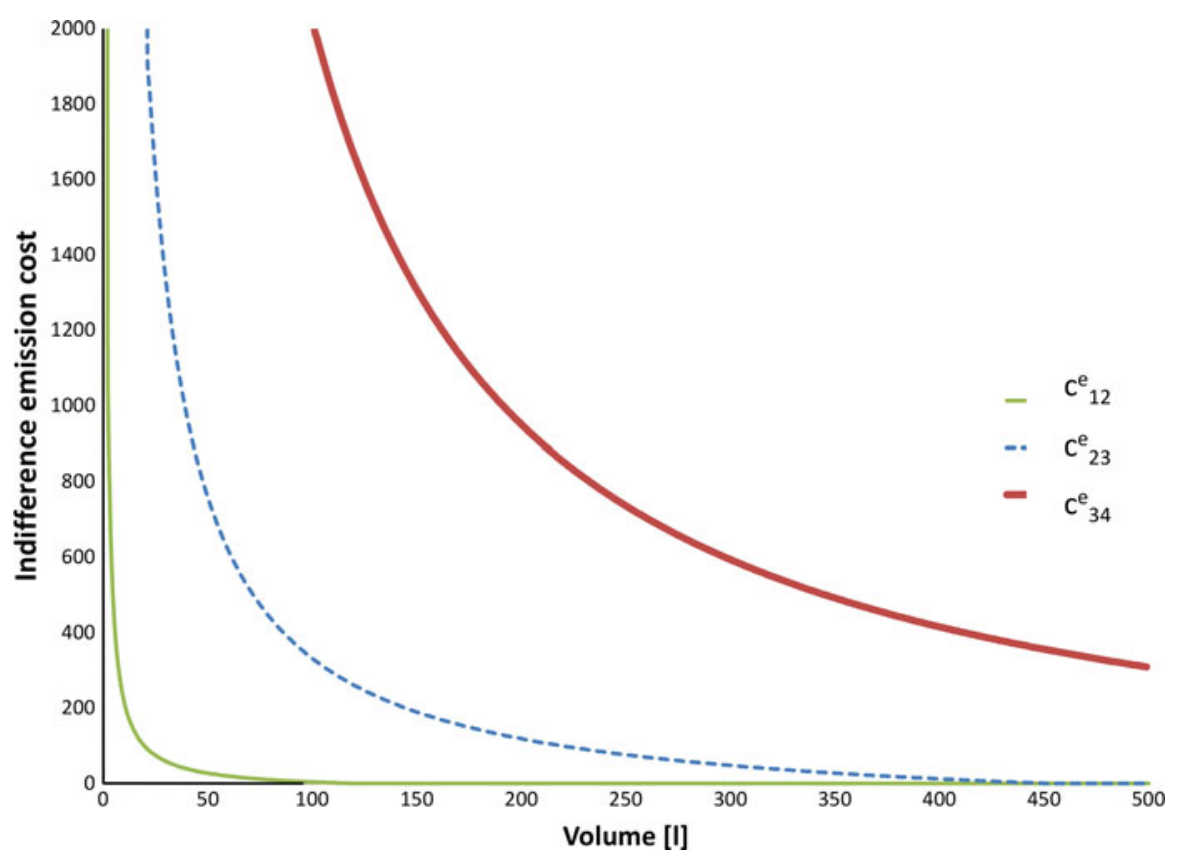

Fig. 1 Indifference emission cost/t $\mathrm{CO}_{2}$ as a function of the product volume

changes in $c^{e}$. This result also has a practical implication for companies: the volume of the packaged product is something that companies can change relatively easy. A decrease in the volume of the packaged product leads to a cost reduction, because both the unit transportation costs and the (allocated) emissions decrease.

A similar graph can be constructed for the distance (Fig. 2). It can be seen that the indifference emission cost is decreasing as a function of distance. The indifference emission cost for road and rail transport is increasing for small distances (up to $85 \mathrm{~km}$ ) due to the small fixed emission factor of road transport. This implies that for larger distances rail and water transport are selected for more values of the emission cost. Air transport is a preferred mode for $d \geq 860$ and selected for very small values of $c^{e}$. This is caused by the fact that the lead time for road transport increases in $d$ (and is constant for air) making the holding and penalty costs for road transport large enough to compensate for lower transport costs. The value of $c_{1,2}^{e}$ remains small due to large unit emissions for air transport.

The emission indifference cost as a function of density is given by Fig. 3. In general, the indifference emission cost is decreasing as a function of density. The indifference cost for road and rail transport is first increasing and then decreasing due to the minimum density for road (transport costs and emissions do not change for $\rho \leq 250$, since $w_{2}$ is constant). Hence, road transport becomes more attractive as the density increases. Air transport is a preferred mode for low values of the density (up to $585 \mathrm{kgm}^{3}$ ) and selected for small values of the emission cost. Note that $c_{1,2}^{e}$ is constant for $\rho \leq 167$, since $w_{1}$ and $w_{2}$ are constant. 


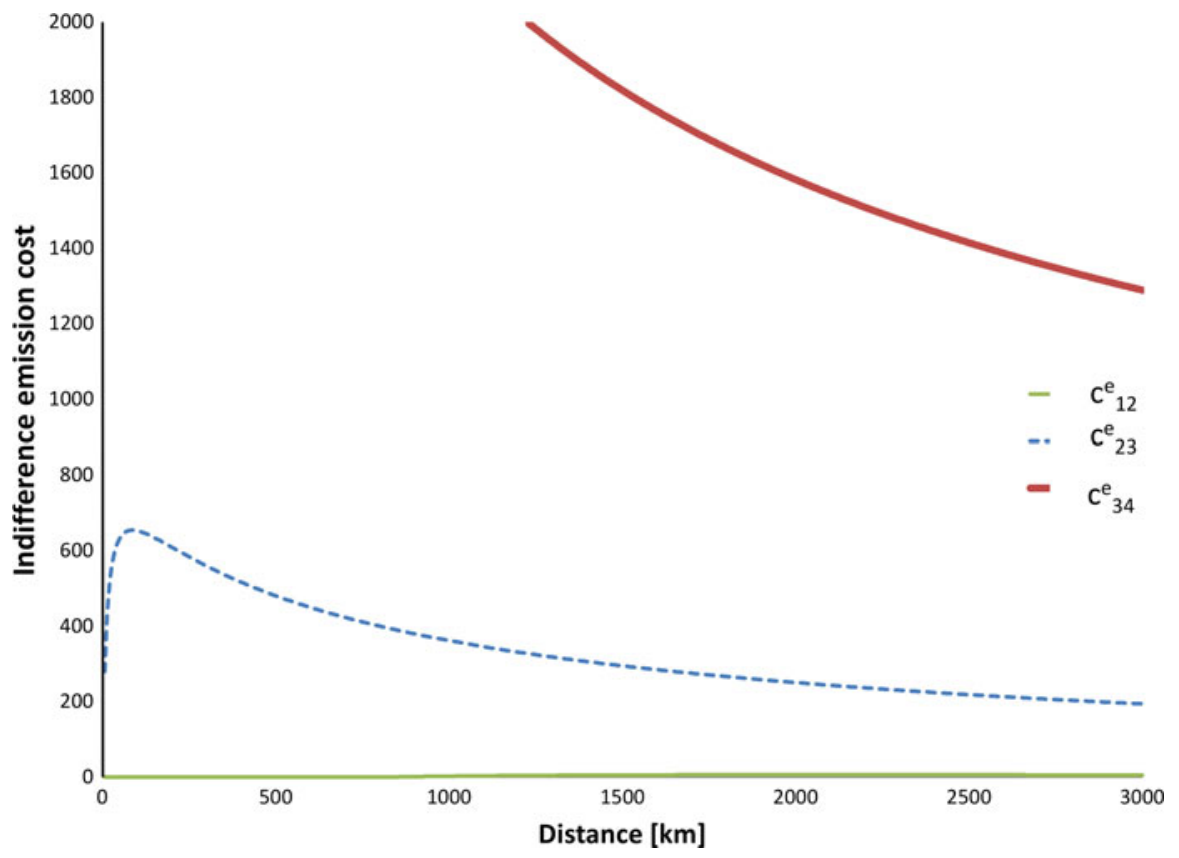

Fig. 2 Indifference emission cost/t $\mathrm{CO}_{2}$ as a function of the distance

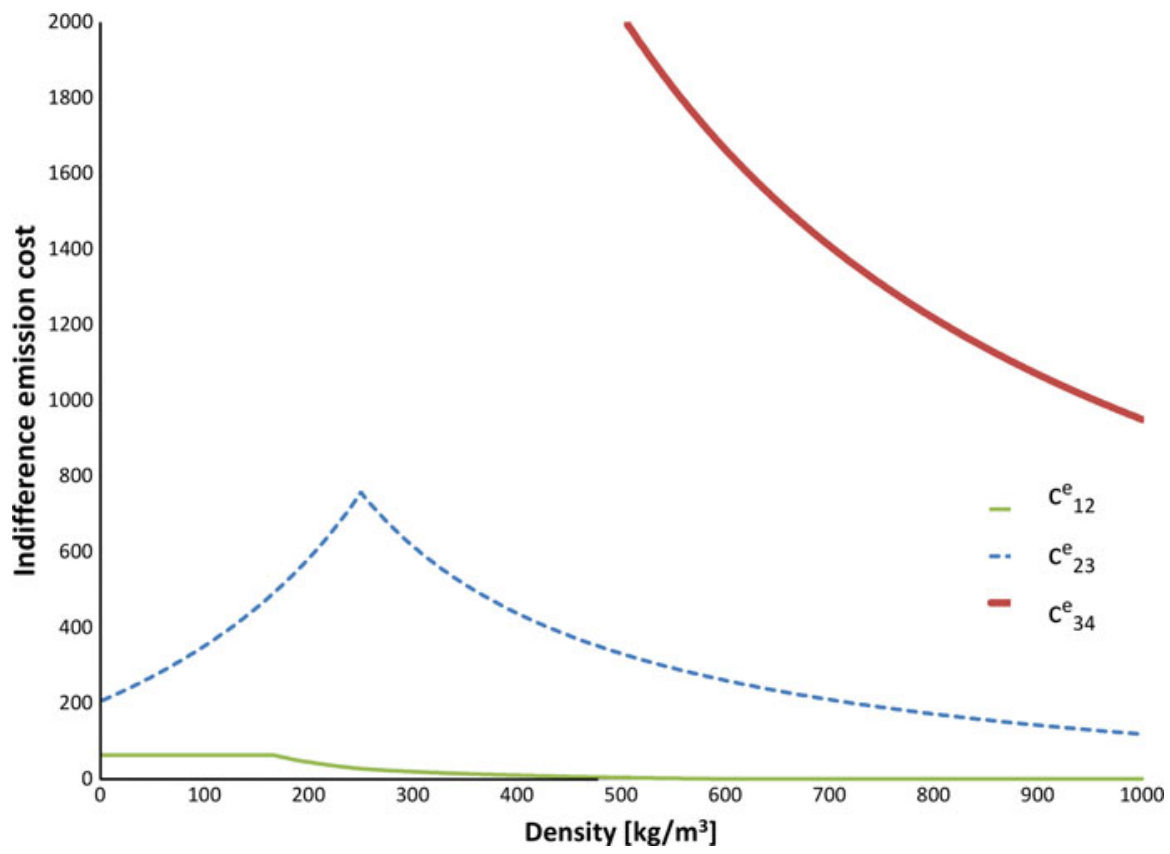

Fig. 3 Indifference emission $\cos \mathrm{t} / \mathrm{t} \mathrm{CO}_{2}$ as a function of the product density 


\subsection{Impact of emission regulation}

In this section we investigate the impact of emission regulations on four particular products. We consider two low-volume products $\left(v=6.4 \cdot 10^{-3}\right)$ : Sugar $(k=€ 1$, and $\rho=1,586)$, Gold (a gold bar) $(k=€ 9,635$, and $\rho=19,320)$, and two high-volume products $\left(v=3.375 \cdot 10^{-1}\right)$ : Insulation material $(k=€ 12.50$, and $\rho=141)$, and a (high-end) Television $(k=€ 4,000$, and $\rho=146)$. We take the same parameter values as before and $d=1,200$. Two products have low value-density $\left(\frac{k}{\rho}\right)$ and the other two have high value-density, one of each with low volume and one with high volume. In Table 5 we present the minimum expected total costs when $c^{e}=0$, the unit emissions and the indifference emission cost value for pairs of modes. In the absence of emission regulation the selected transport modes are water, road, water, and air, for sugar, gold, insulation, and television, respectively. Note that for both Sugar and Insulation no emission reductions are possible by switching transport modes, since water transport minimizes unit emissions. Other emission reduction options have to be explored for those products if emissions need to be reduced. The minimum expected total costs strictly increase in the emission cost, as specified by Proposition 1. For Gold and Television, the higher transport cost of road and air transport is balanced by lower inventory and penalty costs. For Gold the emissions can be reduced by $54 \%$ when a switch is made to rail transport and by $71 \%$ when a switch is made to water transport. For Television the reductions are much higher since air transport is the selected mode when $c^{e}=0,97$ and $98 \%$ for rail and water, respectively.

We conclude this section with an analysis of the selected transport mode for each of the four products for several emission regulation alternatives. First, consider that transport is included in the existing emissions trading scheme and that an emission price of $€ 15 / t$ applies. Since its introduction the price of a carbon allowance has varied between $€ 0$ and $€ 30 / t$ of $\mathrm{CO}_{2}$ (European Carbon Exchange 2011). This would imply that only for television a switch would be made (from air to rail transport), which leads to a significant emission reduction of $97 \%$.

Second, if a carbon tax would apply its value can be much higher than $€ 15 / \mathrm{t}$. Note that the tax should be $€ 237 / t$ to realize emission reductions for Gold. One essential difference between an emissions trading scheme and carbon tax is that a certain amount of allowances may be obtained for free, thereby effectively reducing the carbon cost per unit. Moreover, in an emissions trading scheme the price is uncertain and changes over time.

Third, if a cap would be applied, either voluntarily or by regulation, then emissions reductions are realized for gold and television. If the target was to reduce

Table 5 Total costs, unit emissions and indifference emission cost (in $€ / t$ ) for the four products

\begin{tabular}{lrrrrlllllll}
\hline Product & $C_{1,0}^{*}$ & \multicolumn{1}{c}{$C_{2,0}^{*}$} & \multicolumn{1}{c}{$C_{3,0}^{*}$} & \multicolumn{1}{c}{$C_{4,0}^{*}$} & $e_{1}$ & $e_{2}$ & $e_{3}$ & $e_{4}$ & $c_{1,3}^{e}$ & $c_{2,3}^{e}$ & $c_{3,4}^{e}$ \\
\hline Sugar & 3.05 & 1.53 & 1.23 & 1.11 & 0.0083 & 0.0006 & 0.0003 & 0.0002 & & \\
Gold & 77.98 & 76.36 & 85.64 & 104.75 & 0.1006 & 0.0072 & 0.0033 & 0.0021 & & 237 & 1,547 \\
Insulation & 16.97 & 12.74 & 5.81 & 5.26 & 0.0459 & 0.0049 & 0.0013 & 0.0008 & & \\
Television & 33.88 & 36.66 & 35.30 & 43.27 & 0.0459 & 0.0049 & 0.0013 & 0.0008 & 4 & & 1,617 \\
\hline
\end{tabular}


emissions up to $54 \%$, then rail transport would be selected for Gold and for a target between 54 and $71 \%$, water transport would be selected. The costs increase by 41 and $79 \%$, respectively. For Television a target below $97 \%$ would result in $5 \%$ cost increase. And a target of $98 \%$ emission reduction, would result in $67 \%$ cost increase. Observe that a decreasing rate of return applies in decreasing emissions, i.e. it is increasingly expensive.

For these four products the emission reduction potentials are very different. For two products no reductions can be realized, for one medium reductions and for one very large reductions. If a company would produce these four products and set an emission cap for the joint emissions, then emissions can be reduced by $93 \%$, at a $32 \%$ cost increase. Setting a constraint for a group of items allows the decision maker to reduce emissions substantially where it is cheap, this is called the portfolio effect.

\section{Conclusion}

Since transport emissions account for a substantial share of total carbon emissions, and an even larger share of the expected growth in carbon emissions, policy makers are developing regulation mechanisms that are expected to drive down emissions. Policy makers expect that for instance the transportation mode selection decision will be affected by regulatory frameworks that essentially charge for or limit the emission quantity. It is, however, unclear to what extent emission related costs will play a role in the transportation mode selection problem, since it is obvious that emission costs are only a part of the total costs involved. Therefore, in this paper we have analyzed the effect of (self-imposed) emission regulations on the TMSP in terms of cost and emissions of the solution. Our focus is on a decision maker who has to select one out of several available modes of transportation for a given product. Note that our analysis can be repeated for each product-lane combination, as a 3PL is in charge of transportation and hence there is no significant set-up cost that triggers joint transportation for the shipper. We use an order-up-to policy and the solution of the single-period Newsvendor problem is used to solve the TMSP and yields the optimal expected total cost.

We used the NTM methodology, which is based on empirical data of activities in transportation that cause carbon emissions, to provide formulas and parameter estimates to determine carbon emissions. For our test bed the order of decreasing emissions is: air, road, rail and water. We derived conditions that determine whether a transport is selected for any value of the emission cost. Moreover, we determine which modes are preferred and for which range of the emission cost, given distance, cost and product characteristics, they are selected.

Our numerical results clearly show that the impact of emission related charges is small: the emission related charges or the values of one or more of the parameters (weight, distance or unit cost) needs to be extremely high in order for a decision maker to select a different transportation mode. So, adding emission costs leads rarely to a change in the selected transport mode. This in turn implies that water transport is already the selected mode for many products. In the case of an emission cap, emission reductions are possible but sometimes at a large cost increase. If the company ships a 
heterogenous set of products, in terms of value, density, and volume, then setting an overall cap may lead to emission reductions at smaller cost increases.

In practice, however, other factors play a role as well that make the actual selected mode different from the optimal solution to our problem. For example, water transport may not be available for a certain route or prohibitively expensive. Factors that influence the decision, that we did not take into account are: frequency at which the required mode can be executed for a given route (especially for rail transport), variability of the lead time, and others. Also, we have only calculated the emissions from terminal to terminal and have not considered the distance from the origin and destination to the terminal. For example, water transport does not minimize expected total costs without emissions if the nearest harbor is hundreds of kilometers away from the origin or destination. It may however still result in lower emissions. Extending our model to include one or more of these factors would ensure that the solution of the model coincides more often with the transport mode which is selected in practice.

Open Access This article is distributed under the terms of the Creative Commons Attribution License which permits any use, distribution, and reproduction in any medium, provided the original author(s) and the source are credited.

\section{Appendix 1}

Proofs

\section{Proof of Proposition 1}

Proof of part a The critical fractile $\left(\alpha_{i}\left(c^{e}\right)=\frac{p}{p+\left(k+c_{i}+c^{e} e_{i}\right) r_{h}}\right)$ is decreasing in $c^{e}$. Hence, $S_{i}^{*}\left(c^{e}\right)=F^{-1}\left(\alpha_{i}\left(c^{e}\right)\right)$ is decreasing in $c^{e}$.

Proof of part $b$ Recall that $\mathbb{E}\left[B_{i}^{*}\left(c^{e}\right)\right]=G\left(S_{i}^{*}\left(c^{e}\right)\right)$. Applying the result of part a directly yields that $G\left(S_{i}^{*}\left(c^{e}\right)\right)$ is increasing in $c^{e}$. Hence, $\mathbb{E}\left[B_{i}^{*}\left(c^{e}\right)\right]$ is increasing in $c^{e}$.

Proof of part $c \quad$ Let $S=S_{i}^{*}\left(c^{e}\right)$ and define $H(S)=\mathbb{E}\left[Y^{*}\right]=\int_{-\infty}^{S}(S-y) f(y) \mathrm{d} y$. Let $\epsilon \geq 0$, hence,

$$
\begin{aligned}
H(S+\epsilon)= & \int_{-\infty}^{S+\epsilon}(S+\epsilon-y) f(y) \mathrm{d} y=\int_{-\infty}^{S}(S-y) f(y) \mathrm{d} y+\int_{S}^{S+\epsilon}(S-y) f(y) \mathrm{d} y \\
& +\epsilon \int_{-\infty}^{S+\epsilon} f(y) \mathrm{d} y \geq \int_{-\infty}^{S}(S-y) f(y) \mathrm{d} y .
\end{aligned}
$$

So $H(S)$ is increasing in $S$ and therefore decreasing in $c^{e}$ (using result of part a). Hence, $\mathbb{E}\left[Y_{i}^{*}\left(c^{e}\right)\right]$ is decreasing in $c^{e}$. 
Proof of part $d \quad$ Take $c^{e}$ and $\tilde{c}^{e}=c^{e}+\epsilon$ and $c^{e}, \epsilon \geq 0$.

Recall that $C_{i}\left(S_{i} \mid c^{e}\right)=h_{i}\left(c^{e}\right)\left(S_{i}-\mu_{i}\right)+\left(h_{i}\left(c^{e}\right)+p\right) G_{i}\left(S_{i}\right)+\left(c_{i}+c^{e} e_{i}\right) \mu$.

Let $S_{i} \geq 0$, then $C_{i}\left(S_{i} \mid \tilde{c}^{e}\right) \geq C_{i}\left(S_{i} \mid c^{e}\right)$.

Let $S_{i}^{\epsilon, *}$ minimize $C_{i}\left(S_{i} \mid \tilde{c}^{e}\right)$ and $S_{i}^{*}$ minimize $C_{i}\left(S_{i} \mid c^{e}\right)$.

Then $C_{i}\left(S_{i}^{\epsilon, *} \mid \tilde{c}^{e}\right) \geq C_{i}\left(S_{i}^{\epsilon, *} \mid c^{e}\right) \geq C_{i}\left(S_{i}^{*} \mid c^{e}\right)$ and $C_{i}^{*}\left(\tilde{c}^{e}\right) \geq C_{i}^{*}\left(c^{e}\right)$.

Hence $C_{i}^{*}\left(c^{e}\right)$ is increasing in $c^{e}$.

\section{Proof of Lemma 1}

Proof of part a Assume that $e_{i_{2}} \geq \bar{e}_{i_{2}}\left(i_{1}, i_{3}\right)$.

We need to prove that $C_{i_{2}}^{*}\left(c^{e}\right)>\min \left\{C_{i_{1}}^{*}\left(c^{e}\right), C_{i_{3}}^{*}\left(c^{e}\right)\right\} \forall c^{e} \geq 0$.

We first show that $c_{i_{1}, i_{2}}^{e} \geq c_{i_{1}, i_{3}}^{e}$. Recall that $c_{i_{1}, i_{2}}^{e}=\frac{C_{i_{1}, 0}^{*}-C_{i_{2}, 0}^{*}}{e_{i_{2}}-e_{i_{1}}}$.

$$
\begin{aligned}
-c_{i_{1}, i_{2}}^{e} & =\frac{C_{i_{2}, 0}^{*}-C_{i_{1}, 0}^{*}}{e_{i_{2}}-e_{i_{1}}} \leq \frac{C_{i_{2}, 0}^{*}-C_{i_{1}, 0}^{*}}{\bar{e}_{i_{2}}\left(i_{1}, i_{3}\right)-e_{i_{1}}} \\
& =\frac{C_{i_{2}, 0}^{*}-C_{i_{1}, 0}^{*}}{e_{i_{1}}+\left(e_{i_{3}}-e_{i_{1}}\right) \frac{C_{i_{2}, 0}^{*}-C_{i_{1}, 0}^{*}}{C_{i_{3}, 0}^{*}-C_{i_{1}, 0}^{*}}-e_{i_{1}}}=\frac{C_{i_{3}, 0}^{*}-C_{i_{1}, 0}^{*}}{e_{i_{3}}-e_{i_{1}}} \\
& =-\frac{C_{i_{1}, 0}^{*}-C_{i_{3}, 0}^{*}}{e_{i_{3}}-e_{i_{1}}}=-c_{i_{1}, i_{3}}^{e}
\end{aligned}
$$

(where the inequality follows since $e_{i_{2}}-e_{i_{1}} \leq 0$.)

Next, we show that $c_{i_{1}, i_{3}}^{e} \geq c_{i_{2}, i_{3}}^{e}$.

$$
\begin{aligned}
c_{i_{2}, i_{3}}^{e} & =\frac{C_{i_{2}, 0}^{*}-C_{i_{3}, 0}^{*}}{e_{i_{3}}-e_{i_{2}}} \leq \frac{C_{i_{2}, 0}^{*}-C_{i_{3}, 0}^{*}}{e_{i_{3}}-\bar{e}_{i_{2}}\left(i_{1}, i_{3}\right)} \\
& =\frac{C_{i_{2}, 0}^{*}-C_{i_{3}, 0}^{*}}{e_{i_{3}}-e_{i_{1}}-\left(e_{i_{3}}-e_{i_{1}}\right) \frac{C_{i_{2}, 0}^{*}-C_{i_{1}, 0}^{*}}{C_{i_{3}, 0}^{*}-C_{i_{1}, 0}^{*}}}=\frac{C_{i_{1}, 0}^{*}-C_{i_{3}, 0}^{*}}{e_{i_{3}}-e_{i_{1}}}=c_{i_{1}, i_{3}}^{e} .
\end{aligned}
$$

So we find the following ordering $c_{i_{1}, i_{2}}^{e} \geq c_{i_{1}, i_{3}}^{e} \geq c_{i_{2}, i_{3}}^{e}$, which determines the ordering of the profits for any value of $c^{e}$ :

$$
\begin{array}{ll}
C_{i_{1}}^{*}\left(c^{e}\right) \leq C_{i_{2}}^{*}\left(c^{e}\right) \leq C_{i_{3}}^{*}\left(c^{e}\right) & \text { for } 0 \leq c^{e} \leq c_{i_{2}, i_{3}}^{e} \\
C_{i_{1}}^{*}\left(c^{e}\right) \leq C_{i_{3}}^{*}\left(c^{e}\right) \leq C_{i_{2}}^{*}\left(c^{e}\right) & \text { for } c_{i_{2}, i_{3}}^{e} \leq c^{e} \leq c_{i_{1}, i_{3}}^{e} \\
C_{i_{3}}^{*}\left(c^{e}\right) \leq C_{i_{1}}^{*}\left(c^{e}\right) \leq C_{i_{2}}^{*}\left(c^{e}\right) & \text { for } c_{i_{1}, i_{3}}^{e} \leq c^{e} \leq c_{i_{1}, i_{2}}^{e} \\
C_{i_{3}}^{*}\left(c^{e}\right) \leq C_{i_{2}}^{*}\left(c^{e}\right) \leq C_{i_{1}}^{*}\left(c^{e}\right) & \text { for } c^{e} \geq c_{i_{1}, i_{2}}^{e}
\end{array}
$$

Recall $C_{i_{1}}^{*}\left(c^{e}\right) \leq C_{i_{3}}^{*}\left(c^{e}\right)$ for $c^{e} \leq c_{i_{2}, i_{3}}^{e}$ (and vice versa). From this we can conclude that $C_{i_{2}}^{*}\left(c^{e}\right)>\min \left\{C_{i_{1}}^{*}\left(c^{e}\right), C_{i_{3}}^{*}\left(c^{e}\right)\right\} \forall c^{e} \geq 0$.

Proof of part $b$ Proof is analogous to proof of part a. If $e_{i_{2}} \leq \bar{e}_{i_{2}}\left(i_{1}, i_{3}\right)$, then $c_{i_{1}, i_{2}}^{e} \leq c_{i_{1}, i_{3}}^{e} \leq c_{i_{2}, i_{3}}^{e}$.

The ordering of the profits for any value of $c^{e}$ is: 


$$
\begin{array}{ll}
C_{i_{1}}^{*}\left(c^{e}\right)<C_{i_{2}}^{*}\left(c^{e}\right)<C_{i_{3}}^{*}\left(c^{e}\right) & \text { for } 0 \leq c^{e} \leq c_{i_{1}, i_{2}}^{e} \\
C_{i_{2}}^{*}\left(c^{e}\right)<C_{i_{1}}^{*}\left(c^{e}\right)<C_{i_{3}}^{*}\left(c^{e}\right) & \text { for } c_{i_{1}, i_{2}}^{e} \leq c^{e} \leq c_{i_{1}, i_{3}}^{e} \\
C_{i_{2}}^{*}\left(c^{e}\right)<C_{i_{3}}^{*}\left(c^{e}\right)<C_{i_{1}}^{*}\left(c^{e}\right) & \text { for } c_{i_{1}, i_{3}}^{e} \leq c^{e} \leq c_{i_{2}, i_{3}}^{e} \\
C_{i_{3}}^{*}\left(c^{e}\right)<C_{i_{2}}^{*}\left(c^{e}\right)<C_{i_{1}}^{*}\left(c^{e}\right) & \text { for } c^{e} \geq c_{i_{2}, i_{3}}^{e}
\end{array}
$$

From this we can conclude that $C_{i_{2}}^{*}\left(c^{e}\right)=\min \left\{C_{i_{1}}^{*}\left(c^{e}\right), C_{i_{2}}^{*}\left(c^{e}\right), C_{i_{3}}^{*}\left(c^{e}\right)\right\} \forall c^{e} \in$ $\left[c_{i_{1}, i_{2}}^{e}, c_{i_{2}, i_{3}}^{e}\right]$.

\section{Appendix 2}

Details of NTM emission calculation

In the following sections we describe the unit emission calculation for a particular vehicle for each of the four transport types: air, road, rail, and water, which we will denote by mode $1,2,3$, and 4 , respectively. The traveled distance is expressed as a fraction of the distance for road transport, which is denoted by $d$. The unit emissions are expressed in $\mathrm{kg}$ of $\mathrm{CO}_{2}$ as a function of the distance in $\mathrm{km}$ and the weight of the product in $\mathrm{kg}$.

\section{Air transport}

All assumptions in this section are taken from NTM Air (2008) unless indicated otherwise. The emissions for one unit shipped by an aircraft are determined with the following equation:

$$
e_{1}=\left(E_{1}+\epsilon_{1} \cdot d_{1}\right) \frac{v \max \left\{\rho, \bar{\rho}_{1}\right\}}{\hat{W}_{1} \lambda_{1}} .
$$

Below we describe each of the factors in more detail. We select a Boeing 757-200SF whose emission factors represent a medium-sized aircraft.

Allocation In an applied study (Van den Akker 2009) on logistics service providers it was found that the average load factor for cargo aircrafts is $80 \%$ for dedicated cargo aircrafts, which is the value we assume $\left(\lambda_{1}=0.80\right)$. For the Boeing 757-200SF the maximum load $\hat{W}_{1}$ is $29,029 \mathrm{~kg}$. The minimum density for air transport is $167\left(\bar{\rho}_{1}=167 \mathrm{~kg} / \mathrm{m}^{3}\right)$, which is a density commonly used by transport companies.

Distance The flight distance is calculated with the method used by the International Civil Aviation Organization (ICAO): the Great-circle distance formula. The Great-circle distance formula gives the shortest distance between two locations on a sphere by following a path on the surface of the sphere. For a selected set of routes within Europe the road-distance was compared to the airdistance and it was found that the air distance is $80 \%$ of the road distance on average $\left(d_{1}=0.80 d\right)$.

Emission factors In air transport the constant emission factor corresponds to the emissions during take-off and landing. NTM provides the emission factors for load 
Table 6 Emission factors

\begin{tabular}{lll}
\hline$\lambda_{1}(\%)$ & $E_{1}(\mathrm{~kg})$ & $\epsilon_{1}(\mathrm{~kg} / \mathrm{km})$ \\
\hline 50 & $3,583.901$ & 15.307 \\
75 & $4,041.709$ & 15.351 \\
100 & $4,531.182$ & 15.363 \\
\hline
\end{tabular}

Table 7 Fuel consumption

\begin{tabular}{|c|c|c|c|c|c|c|}
\hline \multirow{2}{*}{$\begin{array}{l}\text { Road type } \\
\text { Load factor }\end{array}$} & \multicolumn{2}{|c|}{ Motor way } & \multicolumn{2}{|l|}{ Rural } & \multicolumn{2}{|l|}{ Urban } \\
\hline & $0 \%$ & $100 \%$ & $0 \%$ & $100 \%$ & $0 \%$ & $100 \%$ \\
\hline Fuel consumption & 0.226 & 0.360 & 0.230 & 0.396 & 0.288 & 0.504 \\
\hline
\end{tabular}

factors of 50, 75 and $100 \%$. The emission factors for different load factors are found by interpolation. and the corresponding emission factors are in Table 6. Using a $80 \%$ load factor, the emission factors are $E_{1}=4,139.6 \mathrm{~kg} \mathrm{CO}_{2}$ and $\epsilon_{1}=15.353 \mathrm{~kg} \mathrm{CO} / \mathrm{km}$.

Unit emissions The unit emissions $\left(e_{1}\right.$ in $\left.\mathrm{kg}\right)$ are then in this case:

$$
\begin{aligned}
e_{1} & =(4,139.6+15.353 \cdot 0.801 d) \frac{v \max \{\rho, 167\}}{23223.2} \\
& \approx v \max \{167, \rho\}\left(1.783 \cdot 10^{-1}+5.295 \cdot 10^{-4} d\right) .
\end{aligned}
$$

\section{Road transport}

All assumptions are taken from NTM Road (2008) unless indicated otherwise. The formula to calculate the unit emissions for road transport $\left(e_{2}\right)$ is:

$$
e_{2}=\left(E_{2}+\epsilon_{2} d_{2}\right) \frac{v \max \left\{\rho, \bar{\rho}_{2}\right\}}{\hat{W}_{2} \lambda_{2}}
$$

We assume that a tractor + semi-trailer is used, because it is a common type to use for longer distances. Moreover, we assume that transport takes place via integrating terminals. Hence, we assume no positioning distance or empty returns.

Allocation We assume a load factor of $70 \%\left(\lambda_{2}=0.70\right)$, which is appropriate for transport via integrating terminals. For a tractor + semi-trailer the maximum load $\hat{W}_{2}$ is $26 \mathrm{t}$. The minimum density for road transport is $250\left(\bar{\rho}_{2}=250 \mathrm{~kg} / \mathrm{m}^{3}\right)$, which is a density commonly used by transport companies.

Distance As mentioned before, the distance for all modality types is expressed in terms of the road distance, hence $d_{2}=d$.

Emission factor The emission factor is determined by the fuel consumption (in $l /$ $\mathrm{km}$ ) and the fuel emissions (in $\mathrm{kg} / \mathrm{l}$ ). The fuel consumption depends on the road type and the values are given in Table 7 . For a load factor of $70 \%$, the fuel consumption is $0.3198,0.3462$ and $0.43921 / \mathrm{km}$, for motorway, rural and urban respectively. For several routes in Europe, we investigated the average distance on urban roads and 
we found it to be $8.9 \mathrm{~km}$ (and in total $17.8 \mathrm{~km}$ for a route). The fuel emissions for diesel fuel are $F E=2.621 \mathrm{~kg} \mathrm{CO}_{2} / 1$. To account for hilly terrain we add $5 \%$ (average value for Europe) to the total emissions. If we implement these values in the formulas for the constant and variable emissions $\left(E_{2}\right.$, in $\mathrm{kg} \mathrm{CO}_{2}$, and $\epsilon_{2}$ in $\mathrm{kg}$ $\left.\mathrm{CO}_{2} / \mathrm{km}\right)$, we obtain:

$$
\begin{aligned}
E_{2}=1.05 \cdot 2.621 \cdot & 17.8(0.4392-0.3198) & \epsilon_{2}=1.05 \cdot 2.621 \cdot 0.3198 \\
& \approx 5.849 & \approx 0.8801 .
\end{aligned}
$$

Unit emissions The unit emissions $\left(e_{2}\right.$ in $\left.\mathrm{kg} \mathrm{CO}_{2}\right)$ are then in this case:

$$
\begin{aligned}
e_{2} & =\frac{v \max \{250, \rho\}}{18,200}(5.849+0.8801 d) \\
& \approx v \max \{250, \rho\}\left(3.214 \cdot 10^{-4}+4.836 \cdot 10^{-5} d\right) .
\end{aligned}
$$

\section{Rail transport}

Two types of rail transport are distinguished; electrical and diesel. All assumptions in this section are taken from NTM Rail (2008) unless indicated otherwise. The emissions for one unit shipped by by rail transport $\left(e_{3}\right)$ are determined with the following equation:

$$
e_{3}=\sum_{z \in Z} \epsilon_{3, j}^{z} d_{3}^{z} w
$$

where the emission factors for electrical $\left(\epsilon_{3,1}^{z}\right)$ and diesel rail $\left(\epsilon_{3,2}^{z}\right)$ in country $z$ are:

$$
\epsilon_{3,1}^{z}=\frac{T\left(\tau^{z}\right) \cdot E E^{z}}{10^{6} \sqrt{W_{g r}} \cdot \lambda_{3,1} \cdot(1-\eta)}, \quad \epsilon_{3,2}^{z}=\frac{T\left(\tau^{z}\right) \cdot F E^{z}}{10^{6} \sqrt{W_{g r}} \cdot \lambda_{3,2}} .
$$

For rail transport, the distance is divided into distances per country because the emissions to generate $1 \mathrm{kWh}$, and the fuel content, differ per country. Define the set of countries through which the train travels as $Z$ and $z \in Z$. In our calculations, we do not distinguish between different countries but assume Europe-averages. We also use the overall shares of electrical and diesel rail: 75.4 and $24.6 \%$, respectively (EUrostat 2010).

Distance The distance traveled in country $z, d_{3}^{z}$, (in $\mathrm{km}$ ) can be calculated from for example the EcoTransIT web page (ECOTransIT 2011). We assume that the rail distance between two locations is equal to the road distance, $d_{3}=d$.

Emission factor (including allocation) Only a variable emission factor is used for rail transport. It depends on the gross weight of the train, an emission constant, a correcting factor for the terrain, the load factor, energy (or fuel) efficiency factor, and a transfer loss (for electrical only).

The gross weight of the train $\left(W_{g r}\right.$ in $\left.\mathrm{t}\right)$ includes the weight of the locomotive and the carriages, and we assume that $W_{g r}=1,000 \mathrm{t}$ which is the average value specified by NTM. The topography of country $z\left(\tau^{z}\right)$ is classified as one of three types $\left(\tau^{z} \in\{\right.$ flat, hilly, mountainous $\}$ ). Let $T\left(\tau^{z}\right)$ define the energy (cq. fuel) consumption for a country with topography type $\tau^{z}$. It holds that T(mountainous $)>T($ hilly $)>T($ flat $)$. We assume that the terrain is hilly. Let $E E^{z}$ denote the 
energy efficiency in country $z$, the emissions for producing $1 \mathrm{kWh}$ of energy, and let $F E^{z}$ denote the fuel emissions in country $z$, the emissions per liter of fuel burnt. For electrical rail, we assume that $T($ hilly $)=675 \mathrm{Wh} / \mathrm{km}$ and $E E=0.41 \mathrm{~kg} \mathrm{CO} C_{2} / \mathrm{kWh}$ (the average emissions to air when generating $1 \mathrm{kWh}$ in Europe). For diesel rail, we assume that $T($ hilly $)=153.08 \mathrm{~g} / \mathrm{km}$, and $F E=3.175 \mathrm{~kg} \mathrm{CO}_{2} / \mathrm{kg}$ diesel. We assume the average value for the load factor of the train (equals the ratio of net and gross weight of the train): $\lambda_{3,1}=\lambda_{3,2}=0.5$. Moreover, a loss of energy $\eta$ (fraction) is taken into account during energy transfer from the power plant to the train $(\eta=0.10)$.

Inserting these parameter values in Eq. (12) yields (in $\mathrm{kg} \mathrm{CO}_{2} /$ net $\mathrm{kg} \mathrm{km}$ ):

$$
\begin{aligned}
& \epsilon_{3,1}=\frac{675 \cdot 0.41}{10^{6} \sqrt{1,000} \cdot 0.5 \cdot 0.9} \approx 1.945 \cdot 10^{-5}, \\
& \epsilon_{3,2}=\frac{153.08 \cdot 3.175}{10^{6} \sqrt{1,000} \cdot 0.5} \approx 3.074 \cdot 10^{-5} .
\end{aligned}
$$

We combine these emission factors and the average share of electrical and diesel, to obtain the rail emission factor $\epsilon_{3}$ (in $\mathrm{kg} \mathrm{CO} /$ net $\mathrm{kg} \mathrm{km}$ ):

$$
\epsilon_{3}=(0.754 \cdot 1.945+0.246 \cdot 3.074) 10^{-5} \approx 2.223 \cdot 10^{-5} .
$$

Unit emissions The unit emissions ( $e_{3}$ in $\mathrm{kg} \mathrm{CO}_{2}$, where $d$ in $\mathrm{km}$ and $w$ in $\mathrm{kg}$ ) are then in this case:

$$
e_{3} \approx 2.223 \cdot 10^{-5} \cdot d \cdot w
$$

\section{Water transport}

Water transport covers short-sea transport and inland transport with diesel oilpowered vessels. All details are taken from NTM Water (2008). The formula to calculate the unit emissions for road transport $\left(e_{4}\right)$ is:

$$
e_{4}=\beta_{4} \cdot F C_{4} \cdot F E_{4} \cdot d_{4}
$$

where $\beta_{4}=\frac{\text { unitcapacity }}{\text { total capacity }}$ The unit of capacity is dependent on the type of ship used, it can be weight (for bulk vessels), TEU (20-foot equivalent units) (for container vessels) or lane meters (for roll-on, roll-off vessels, which transport trucks or rail carts). We assume that inland waterways are used and select a general cargo vessel. Inland waterways is selected because it covers a wider range of ports in Europe than short-sea. For the selected vessel, allocation is done based on weight: $\beta_{4}=\frac{w}{\hat{W}_{4} \lambda_{4}}$.

Allocation The cargo capacity (maximum load) of a general cargo vessel for inland waterways is $3,840 \mathrm{t}\left(\hat{W}_{4}=3,840 \mathrm{t}\right)$. We assume a load factor of $50 \%$ $\left(\lambda_{4}=0.50\right)$. This factor is relatively low since in inland waterways the transport is shuttle-like.

Emission factor The emission factor is determined by the fuel consumption $(F C)$ and the fuel emissions $(F E)$, which are for the selected vessel $0.007 \mathrm{t} \mathrm{diesel} / \mathrm{km}$, and $3,178 \mathrm{~kg} \mathrm{CO}_{2} / \mathrm{t}$ diesel, respectively. 
Distance The distance $d_{4}$ in $\mathrm{km}$ for short-sea water transport can be obtained from, for example the PortWorld Distance calculator web site (PortWorld Distance Calculator 2011). We assume that the distance between two locations over inland waterways is 1.2 times the distance over road: $d_{4}=1.2 \mathrm{~d}$. Due to a lack of empirical data this value is an educated guess.

Unit emissions Applying these values, yields the following equation for the unit emissions for water transport $\left(e_{4}\right.$ in $\left.\mathrm{kg}\right)$ :

$$
e_{4}=\frac{w}{1,920 \cdot 1,000} 0.007 \cdot 1.20 d \cdot 3,178 \approx 1.3904 \cdot 10^{-5} \cdot w \cdot d .
$$

\section{References}

Atasu A, Guide VDR Jr, Van Wassenhove LN (2008) Product reuse economics in closed-loop supply chain research. Prod Oper Manag 17:483-496

Axsäter S (2006) Inventory control. Springer, Berlin

Bauer J, Bektaş T, Crainic T (2009) Minimizing greenhouse gas emissions in intermodal freight transport: an application to rail service design. J Oper Res Soc 61:530-542

Baumol W, Vinod H (1970) An inventory theoretic model of freight transport demand. Manag Sci 16:413-421

Benjaafar S, Li Y, Daskin M (2010) Carbon footprint and the management of supply chains: insights from simple models. Working paper

Blauwens G, Vandaele N, Vande Voorde E, Vernimmen B, Witlox F (2006) Towards a modal shift in freight transport? A business logistics analysis of some policy measures. Transp Rev 26:239-251

Blumberg D (2005) Introduction to management of reverse logistics and closed loop supply chain processes. CRC Press, Boca Raton, FL

Bonney M, Jaber MY (2011) Environmentally responsible inventory models: non-classical models for a non-classical era. Int J Prod Econ 133:43-53

Corbett C, Kleindorfer P (2001a) Environmental management and operations management: introduction to part 1 (manufacturing and eco-logistics). Prod Oper Manag 10:107-111

Corbett C, Kleindorfer P (2001b) Environmental management and operations management: introduction to part 2 (integrating operations and environmental management systems). Prod Oper Manag 10:225-227

ECOTransIT (2011) Make your own calculation. http://www.ecotransit.org

European Carbon Exchange (2011) ICE products. http://www.ecx.eu

European Commision (2007) European energy and transport trends to 2030 - update 2007. Technical report. European Commision, Directorate-General for Energy and Transport

European Commission (2008) Questions \& answers on emissions trading and national allocation plans. http://europa.eu/rapid/pressReleasesAction.do?reference=MEMO/08/35

European Commission (2012) Reducing emissions from the aviation sector. http://ec.europa.eu/ clima/policies/transport/aviation

EUrostat (2010) Train-movements, by type of vehicle and source of power. http://epp.eurostat. ec.europa.eu/portal/page/portal/transport/data, figure based on year 2005

Fleischmann M, Bloemhof-Ruwaard JM, Dekker R, van der Laan E, van Nunen JAEE, Van Wassenhove LN (1997) Quantitative models for reverse logistics: a review. Eur J Oper Res 103:1-17

Greenhouse Gas Protocol (2011) Greenhouse Gas Protocol. http://www.ghgprotocol.org

Gupta S, Lambert A (2008) Environment conscious manufacturing. CRC Press, Boca Raton, FL

Hua G, Cheng TCE, Wang S (2011) Managing carbon footprints in inventory control. Int J Prod Econ 132:178-185

IPCC (1995) Summary for policymakers: the science of climate change-IPCC Working Group I. http://www1.ipcc.ch/ipccreports/assessments-reports.htm

Kiesmüller GP, de Kok AG, Fransoo JC (2005) Transport mode selection with positive manufacturing lead time. Transp Res Part E 41:511-530

Kleindorfer P, Singhal K, Van Wassenhove L (2005) Sustainable operations management. Prod Oper Manag 14:482-492

NTM (2011) NTM calc. http://www.ntmcalc.se/index.html 
NTM Air (2008) Environmental data for international cargo and passenger air transport. Technical report. NTM. URL: http://www.ntm.se

NTM Rail (2008) Environmental data for international cargo transport. Technical report. NTM. URL: http://www.ntm.se

NTM Road (2008) Environmental data for international cargo transport road transport. Technical report. NTM. URL: http://www.ntm.se

NTM Water (2008) Environmental data for international cargo sea transport. Technical report. NTM. URL: http://www.ntm.se

OECD (2002) Strategies to reduce greenhouse gas emissions from road transport: analytical methods. http://www.internationaltransportforum.org/Pub/pdf/02GreenhouseE.pdf

Pindyck R, Rubinfeld D (2008) Microeconomics, 7th edn. Prentice Hall, Upper Saddle River, NJ

Pochampally KK, Nukala S, Gupta S (2009) Strategic planning models for reverse and closed-loop supply chains. CRC Press, Boca Raton

PortWorld Distance Calculator (2011) Ship voyage distance calculator. http://www.portworld.com/map/

Rosič H, Jammernegg W (2010) The economic and environmental performance of dual sourcing. Working paper. Vienna University of Economics and Business, Austria

Sasikumar P, Kannan G (2009) Issues in reverse supply chain, part III: classification and simple analysis. Int J Sustain Eng 2:2-27

Srivastava SK (2007) Green supply-chain management: a state-of-the-art literature review. Int J Manag Rev 9:53-80

Tyworth JE (1991) The inventory theoretic approach in transportation selection models: a critical review. Logist Transp Rev 27:299-318

Van den Akker IJG (2009) Carbon regulated supply chains: calculating and reducing carbon dioxide emissions for an eye health company. Master's thesis, Eindhoven University of Technology

\section{Author Biographies}

K. M. R. Hoen is a $\mathrm{PhD}$ student at School of Industrial Engineering at Eindhoven University of Technology. In 2008 she obtained her master's degree in Operations Management \& Logistics from Eindhoven University of Technology. Her research interests include sustainable/green supply chain management, inventory theory and transportation.

T. Tan is an Assistant Professor in the School of Industrial Engineering at Eindhoven University of Technology, The Netherlands. Dr. Tan received his PhD in Industrial Engineering from the Middle East Technical University, Ankara, Turkey, in 2002. He pursued one year of his studies towards his $\mathrm{PhD}$ degree at Columbia University, Graduate School of Business, Management Science/Operations Research division, New York, as a Fulbright scholar. He joined Eindhoven University of Technology as a post-doc researcher in 2003 and started working as an assistant professor the same year in the Operations, Planning, Accounting, and Control group. He has recently spent an academic term at the University of California, Los Angeles, as a visiting scholar. Dr. Tan is an executive board member of the European Supply Chain Forum. His research interests include inventory theory, capacity management, spare parts management, and supply chain management with a particular focus on the effects of carbon emissions.

J. C. Fransoo is a Professor of Operations Management and Logistics in the School of Industrial Engineering at Eindhoven University of Technology in the Netherlands. He holds an MSc in Industrial Engineering and $\mathrm{a} \mathrm{PhD}$ in Operations Management \& Logistics from Eindhoven University of Technology. He currently serves as Associate Editor of Operations Research, Senior Editor of Production and Operations Management and is editorial board member of seven other journals. He has published over 60 papers in academic journals and presented at many academic and industry conferences, on topics such production planning, supply chain and inventory management, and transportation planning, with a focus on the chemical, pharmaceutical and food industries. His most recent work is addressing issues such as synchromodal transportation, distribution in megacities, and carbon-efficient supply chain management. 
G. J. van Houtum is Professor of Maintenance, Reliability, and Quality at Eindhoven University of Technology since 2008. Prior to that he filled positions as assistant/associate professor at the University of Twente (1994-1998) and Eindhoven University of Technology (1999-2007). He obtained his MSc and $\mathrm{PhD}$ degree in Applied Mathematics from Eindhoven University of Technology in 1990 and 1995, respectively. He is the scientific director of the Beta Research School for Operations Management and Logistics. His research is focused on: (1) Spare parts management; (2) Maintenance and availability management of capital goods; (3) The effect of design decisions on the total cost of ownership of capital goods. A significant part of this research is in collaboration with companies such as ASML, DAF, IBM, Nedtrain, Océ Technologies, Marel Stork, and Vanderlande Industries. 